\title{
BIOLOGIE DE HYALOMMA DROMEDARII (KOCH 1844)
}

\author{
Par L. DELPY et S.-H. GOUCHEY
}

La biologie des Hyalomma est mal connue. Les rares travaux publiés (Lounsbury, 1900 et 1903 ; Nuttall, 1913 ; Brumpt, 1921 ; E.-A. Lewis, 1932) ainsi que les divers traités, ne font allusion qu'à Hyalomma aegyptium Linné. On sait maintenant que $H$. aegyptium L. n'est autre que $H$. syriacum Koch, tique de la tortue et quand un auteur désigne sous le nom $H$. aegyptium $\mathrm{L}$. une tique des mammifères, il est tout à fait impossible de savoir à quelle espèce de $\mathrm{Hya}$ lomma il fait allusion. Ainsi, Nuttall (1913) désigne par le nom $H$. aegyptium, des tiques trouvées sur des bœufs, chèvres, moutons, autruches en Afrique du Sud, des tiques du mouton d'Algérie, des tiques des bœufs des environs de Rome et des tiques des chameaux de Biskra. Pour E.-L. Lewis (1932), H. aegyptium est la même tique que $H$. impressum albiparmatum Sch. et Schl. Pour Brumpt (Précis, 1936) c'est de $H$. marginatum Koch que $H$. aegyptium L. est synonyme.

C'est à Sergent, Donatien, Parrot et Lestoquard (1931) que nous devons les premiers renseignements sur la biologie d'une espèce déterminée de Hyalomma. Il s'agit de $H$. mauritanicum Senevet, tique dont les mœurs sont assez particulières.

\section{Méthode de travail}

1. Terminologie. - Durée réelle du cycle: nombre de jours du début à la fin du cycle.

Durée corrigée: Durée réelle dont on a soustrait les temps perdus.

Temps perdus : Nombre de jours où les larves, nymphes et adultes ont été conservées à jeun, avant de les placer sur un hôte.

Sommeil larvaire, nymphal : Période qui sépare la fin du repas de la larve ou de la nymphe, de leur mue.

Gestation: Période qui sépare la fin du repas de la femelle du début de la ponte.

Incubation: Période qui sépare le début de la ponte du début de l'éclosion.

Annales de Parasitologie, $\mathrm{T} . \mathrm{XV}, \mathrm{N}^{3} 6 .-1^{\text {er }}$ novembre 1937 , p. $487-499$. 
Génération : Ensemble des individus nés d'une même mère.

Famille: Ensemble des générations provenant d'une même femelle-ancêtre.

2. Gonstitution de familles pures. - La détermination des femelles gorgées, qui doivent être à l'origine de chaque famille, est d'abord faite à titre provisoire d'après la morphologie des femelles, puis confirmée ou rectifiée, en fin de cycle, par l'examen des mâlesfils. Si l'on se fait envoyer de l'extérieur des femelles gorgées, il n'en faut placer qu'une seule par tube, afin que les œufs ne se mélangent pas, si la ponte commence pendant le transport.

3. La figure 5 montre le dispositif que nous employons pour élever les Hyalomma. Ce dispositif convient bien aussi pour les Boophllus et Rhipicephalus. Les autres genres ont des exigences plus précises, en ce qui concerne l'humidité et la température, et doivent être élevés dans d'autres conditions.

4. Repas. - Les larves, nymphes ou adultes, sont placés dans des sacs de toile forte que l'on fixe aux oreilles ou au scrotum des animaux. Lorsqu'on conduit simultanément plusieurs élevages de tiques à 3 hôtes il faut observer strictement un certain nombre de précautions : vérifier que l'hôte ne porte pas de tiques "sauvages » lorsqu'il reçoit un lot d'éleyage; numéroter attentivement les sacs et ne manipuler qu'un sac en même temps ; faire bouillir les sacs après usage, afin d'éviter que des larves ou nymphes se cachent dans les plis et soient ensuite trouvées dans un autre lot.

Enfin, comme malgré toutes les précautions il faut toujours s'attendre à des échecs, ne pas disposer en une seule fois de la totalité des individus d'un lot. Par exemple, si l'on obtient 1.000 larves d'une même mère, ne les mettre à gorger que par lots de 100 , et si possible sur des animaux et à des moments différents : au cas où les larves ne se fixeraient pas, ou bien si le sac se détache de l'hôté, l'élevage n'est pas définitivement perdu.

5. Gonduite des élevages. - On peut agir sur l'évolution des tiques de plusieurs façons : en particulier il est aisé de prolonger la durée du cycle dans des proportions importantes en gardant les larves, nymphes et adultes à jeun, au lieu de les faire gorger aussitôt après la mue. Il est naturel de chercher à déterminer la durée minima et la durée maxima de l'évolution. Dans le premier cas, on fera gorger les tiques immédiatement après chaque mue : dans le second cas, le lot ne sera porté sur l'hôte qu'au moment où on constatera un 
début de mortalité. L'expérience montre que ces tentatives échouent assez souvent, surtout lorsqu'on cherche à prolonger la durée du cycle.

\section{Biologie de Hyalomma dromedarii (Koch 1844)}

1. Hôtes naturels. - Nous n'avons jamais trouvé de larves, à jeun ou gorgées, sur des animaux domestiques ou sauvages. Les nymphes se trouvent sur les chameaux, les bovins et les lièvres. Les adultes se trouvent le plus souvent sur le chameau, parfois sur les bovidés, exceptionnellement sur les autres animaux.

Ces renseignements ont naturellement un caractère provisoire.

2. Hôtes en élevage artificiel. - Il est très difficile d'entretenir au laboratoire un nombre important de chameaux. D'autre part, en Iran, les hérissons meurent très vite en captivité, ainsi que les lièvres. Nous utilisons donc les veaux, moutons, et lapins.

Les larves se gorgent volontiers sur lapin et sur veau, difficilement et rarement sur chameau et mouton. II en est de même pour les nymphes, si ce n'est qu'elles se fixent bien sur le chameau.

Les adultes se fixent rapidement sur le chameau et les bovidés, très rarement sur le mouton.

Il est parfois difficile d'élever sur veau la descendance d'une femelle recueillie sur un chameau, mais nous avons à plusieurs reprises constaté une véritable adaptation. L'observation suivante est assez démonstrative :

Lot $X-179$ : Femelles gorgées, recueillies sur un chameau de caravane et provisoirement déterminées : $H$. dromedarii. Dimensions : $0,20 \times 0,10$ à $0,12 \mathrm{~mm}$.

Femelle $X-179,3$ : Les larves issues de cette famille se fixent très mal sur scrotum de veau. On n'obtient que 10 nymphes de petite taille et, finalement, 6 femelles et 4 mâles. Les mâles sont nettement atypiques : très petits ( 3,3 à $4 \times 2$ à $2,5 \mathrm{~mm}$.), pattes grêles et claires, corps globuleux, voussé, scutum profondément sculpté.

Le seul caractère spécifique net est constitué par la position des écussons subanaux, simples ou doubles, mais dont l'axe est excentrique à l'axe des écussons anaux. Les femelles, très frêles, ont des pattes très minces et jaune clair. Leur capitulum et leur scutum sont normaux.

Femelle $X-179,3,2$ (fille de la précédente). Des œufs de cette femelle on ne réussit à obtenir que 50 larves gorgées. Les nymphes se fixent, mais se détachent avant réplétion. Placées sur un autre veau, certaines se fixent de nouveau, et on en récolte finalement 11 . 
Ces 11 nymphes donnent 7 femelles et 4 mâles. Les mâles sont un peu plus grands que ceux de la génération précédente $(3$ à $5 \times 1,8$ à $3 \mathrm{~mm}$.), mais ont le même aspect voussé et globuleux.

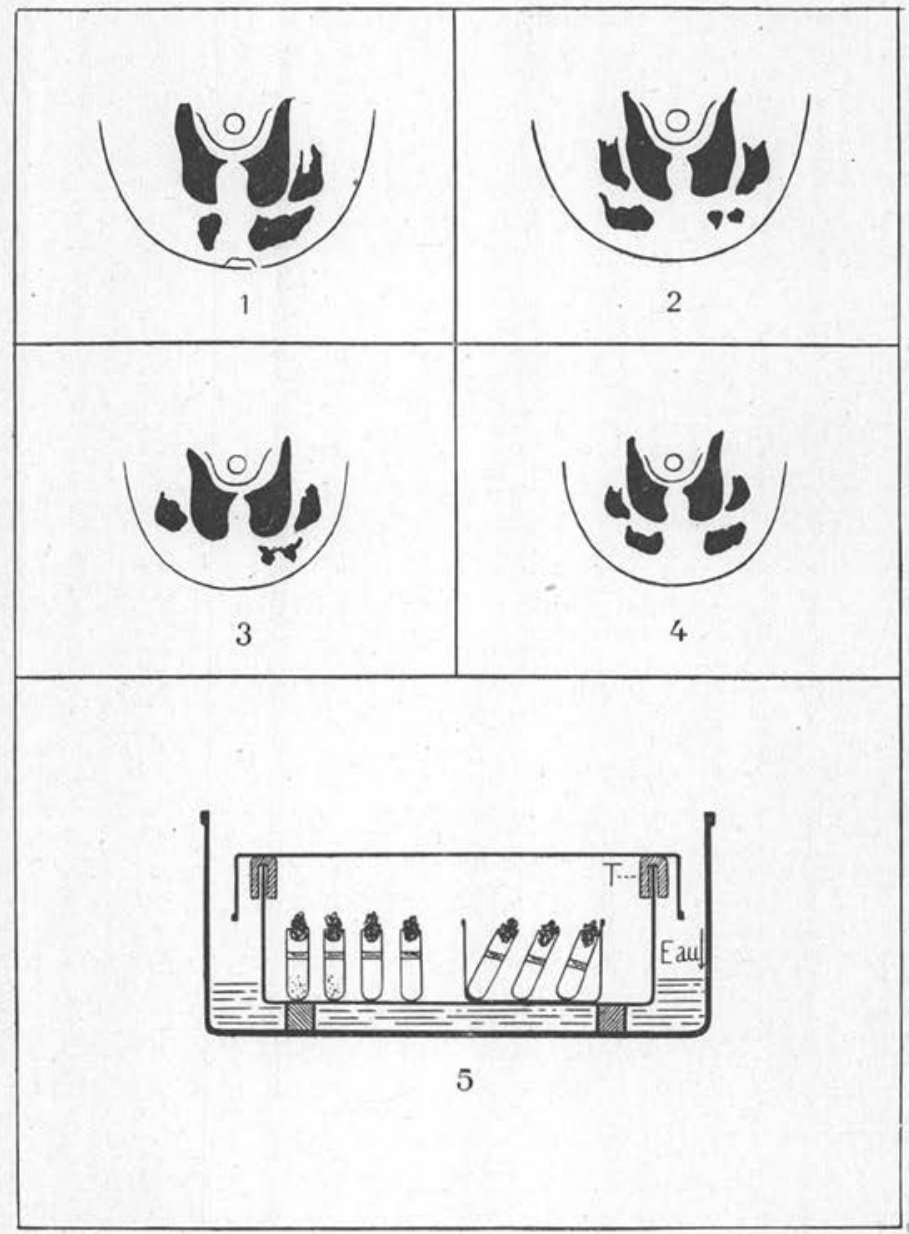

FIG. - 1, 2, 3, 4, Variations des écussons de l'anus chez des mâles de la fam. X-179; 5, Dispositif pour l'élevage des Hyalomma; T, taquets de liège.

L'un d'eux $(3 \times 1,8 \mathrm{~mm}$. $)$ ne présente que deux écussons anaux, sans adanaux ni subanaux. Les femelles sont à peu près normales, mais leurs pattes sont anormalement claires et grêles.

Femelle $X-179,3,2,4$ (fille de la précédente). 
Partant d'environ 100 larves, nous obtenons, toujours sur veau, 75 nymphes, puis 30 mâles et 30 femelles, très bien venus et présentant tous les caractères de $H$. dromedarii. Au nombre de ces caractères, figurent les variations habituelles des écussons subanaux : dans plusieurs cas, un des subanaux manquait; dans deux cas, les deux subanaux étaient absents. Dans un cas, la moitié gauche du système anal était normale, tandis qu'à droite. l'adanal était absent et le subanal sous l'anal (fig. 1 à 4 ).

Avec cette $3^{\circ}$ génération semble s'être terminée la période d'adaptation au veau : la famille X-179 s'élève actuellement sur cet animal sans aucune difficulté.

Remarquons à ce sujet que, d'une façon générale, un élevage ne donne des adultes normaux et vigoureux que si la femelle mère, les larves et les nymphes se sont bien et complètement gorgées.

Il est possible d'obtenir, presque à coup sûr, des spécimens rabougris et anormaux en interrompant le repas de la femelle des larves ou des nymphes. Les «Kummerform » de Schülze, ou «forma miresa » de Tonelli-Rondelli (1932), trouvés sur des chameaux, sont probablement des $H$. dromedarii dont certaines périodes de gorgement ont été insuffisantes.

3. Nombre d'hôtes. - Pendant quatre ans, nous avons vu H. dromedarii évoluer régulièrement sur 3 hôtes. En 1935-1936 deux élevages se sont comportés différemment, et nous résumons leur histoire :

a) La femelle $A-5$ commence à pondre le 24 mai. Les œufs éclosent en juillet, et on place environ 1.000 larves sur le scrotum d'un veau. 200 de ces larves se gorgent et se détachent le $3^{\circ}$ jour, 500 le $5^{\circ}$ jour, mais 150 ont mué sur le testicule même, se sont refixées, et ont fait leur repas nymphal à quelques millimètres de l'endroit où avait eu lieu le repas larvaire. Le $12^{\circ}$ jour, ces nymphes repues se détachaient.

b) Dans le cas de la famille $Z-89$, nous avons deux générations. La première (femelle Z-89) fut gorgée sur lapin à l'état larvaire et nymphal, et sur veau à l'état adulte. Les formes jeunes évoluèrent en hiver. Il y eut 3 hôtes.

La deuxième génération (femelle Z-89-1) débuta en juin par la ponte et les larves furent placées fin août sur lapin. 5 jours plus tard 20 larves gorgées se détachent. Du $6^{\circ}$ au $8^{\circ}$ jour, 43 nymphes à jeun provenant de larves qui ont mué sur place se détachent à leur tour. Enfin, le $12^{\circ}$ jour, 11 nymphes gorgées sont récoltées, provenant de larves qui ont mué sans se détacher. 
Il est à remarquer que, dans ces deux cas, nous ne trouvons qu'un facteur commun : l'époque de l'année où se situe la mue larve-nymphe. Il s'agit de l'été 1936, particulièrement sec et chaud à Hessarek.

Or, en été, les nymphes à jeun sont particulièrement fragiles : de la famille A-5, nous avions gardé à $30^{\circ}$, environ 200 larves à jeun. Après 50 jours, elles étaient encore vivantes. Au contraire, dés nymphes à jeun, gardées dans les mêmes conditions, moururent en moins d'un mois.

En ce qui concerne la famille Z-89, nous voyons, qu'en saison froide, larves et nymphes évoluent sur des hôtes différents. Au mois d'août, au contraire, c'est sur un seul hôte et très rapidement (12 jours) que certaines tiques se gorgent, à l'état larvaire, muent, et se gorgent à l'état nymphal.

Nous sommes portés à voir dans ces évolutions accélérées un phénomène de défense. Les nymphes à jeun tombées sur le sol ont certainement peu de chances de trouver en peu de temps un hôte convenable. Au contraire, si la mue et le repas nymphal se produisent sur l'hôte qui a fourni le repas larvaire, la tique tombera sur le sol à un moment de son existence où elle est relativement très robuste. Ainsi, sont considérablement accrues les probabilités de fermeture du cycle.

En 1913, Nuttall avait observé des phénomènes analogues en gorgeant des Hyalomma sur des hérissons. Il en avait conclu que les larves gorgées restaient empêtrées dans les piquants de l'hôte et ne pouvaient tomber sur le sol : par conséquent, d'après l'auteur anglais, le fait que certaines nymphes se gorgent sur le même hôte que les larves n'a aucune signification biologique. Nos observations montrent que la nature du pelage de l'hôte est sans importance : sur lapin comme sur veau, on observe des évolutions à 3 et 2 hôtes. Il ne peut naturellement être question de contrainte mécanique, empêchant les larves gorgées de se détacher, puisque certaines se détachent, d'autres muent sur place avant de se détacher, mais ne se gorgent pas à l'état nymphal et les dernières, enfin, muent étant fixées, dégagent leur rostre de la plaie, et, nymphes, se gorgent à proximité.

Cependant, il faut souligner le fait que la nymphe ne se gorge pas dans la même blessure que la larve, et ceci différencie ces Hyalomma des Boophilus, par exemple, dont le rostre reste fixé au même endroit pendant le repas de la larve, de la nymphe et parfois de l'adulte.

Hyalomma dromedarii est donc une tique à trois hôtes, ou, si l'on préfère, une tique qui se détache de son hôte entre chacun de ses repas. Toutefois, il arrive que la mue larve-nymphe se produise très vite et que la nymphe se gorge sur l'animal où elle a pris son repas larvaire, L'évolution se fait alors sur deux hôtes seulement. 
Ajoutons que cette dérogation à la règle ne se produit que dans un pour cent des cas, environ et, d'après notre expérience personnelle, lorsque la saison, chaude et sèche, est peu favorable à la vie des nymphes à jeun.

4. Influence de la température et de l'humidité. - Hyalomma dromedarii est relativement très tolérante. Elle résiste bien au froid, et à la chaleur, dans les limites des variations naturelles et se contente d'une faible humidité. Les larves à jeun, mais surtout les nymphes à jeun, sont fragiles et craignent la sécheresse. Quant aux autres formes, elles peuvent résister des mois à des températures voisines de zéro degré, aussi bien qu'à plus de $37^{\circ}$. Ajoutons que, depuis le printemps jusqu'au début de l'hiver, on peut trouver sur les chameaux de caravane des nymphes gorgées et des adultes à jeun ou repus.

Nous verrons, en étudiant la durée du cycle évolutif, qu'elle est variable selon les saisons : en saison chaude, le cycle est plus court qu'en saison froide. Cependant, étant donné les conditions de nos élevages, les tiques sont soumises, hiver comme été, à peu près aux mêmes températures : en été le laboratoire est à $26-33^{\circ}$, et en hiver, les tiques sont maintenues à $20-25^{\circ}$, sauf, bien entendu pendant les repas.

5. Influence de l'accouplement. - Les mâles s'accouplent aux femelles à jeun aussi bien qu'à celles qui sont gorgées, et in vitro aussi bien que sur l'hôte. Le phénomène est facile à observer en mettant en présence une femelle gorgée (par conséquent peu mobile) et quelques mâles. Ces derniers font preuve d'une activité désordonnée et assaillent la femelle jusqu'au moment où l'un d'eux a réussi à s'accoupler.

Il est certain que les femelles ne se gorgent bien que si des mâles ont été placés dans le même sac. A maintes reprises, nous avons observé qu'en l'absence de mâles, elles se détachent prématurément, ou bien restent fixés très longtemps ( 2 mois), sans atteindre pour cela un état normal de réplétion.

Le plus souvent, ces femelles meurent sans avoir pondu.

6. Nombre d'œufs. - Très variable. Varie entre 2.000 et 8.000 environ.

7. Agglomération des larves. - Quelques jours après l'éclosion (le nombre de jours varie avec les saisons et les élevages), les larves qui étaient dispersées le long des parois du tube, se réunissent, s'agglo- 
mèrent et forment une sorte d'essaim, difficile à dissocier. Cet essaim reste compact si on le saisit avec une pince ou si on agite le tube.

A ce moment, les larves se fixent très rapidement, si un hôte leur est offert. Si on attend quelques jours, l'essaim se désagrège, et la fixation des larves devient aléatoire.

\section{Etude détaillée du cycle évolutif}

Rien ne nous permet d'avoir une idée suffisamment précise du cycle évolutif de $H$. dromedarii, dans les conditions naturelles. Le chameau qui est l'hôte le plus fréquent pour la nymphe et l'adulte, se déplace constamment et sur de longs parcours. On peut donc imaginer qu'une jeune femelle qui s'est fixée sur un chameau lors de son passage dans une vallée chaude, se détachera deux semaines plus tard, à un moment où le chameau sera parvenu à un col élevé, au voisinage des neiges...

Le détail des cycles évolutifs « naturels » doit donc être extrêmement varié, et d'ailleurs, ainsi que nous l'avons déjà signalé, on trouve en toute saison, sauf en plein hiver, des nymphes et des adultes sur les grands animaux, particulièrement sur les chameaux.

$\mathrm{Au}$ laboratoire, nous avons cherché à réaliser le cycle le plus court et le cycle le plus long. En ne retenant que la durée corrigée de ces cycles, et en faisant intervenir ce que nous savons de la résistance des larves, nymphes et adultes à jeun, nous avons obtenu des chiffres extrêmes très éloignés les uns des autres, et qui nous indiquent dans quelles limites peut varier la durée des cycles « naturels 》.

Le cycle le plus court que nous ayons obtenu a duré 93 jours, avec 6 jours de temps perdu. La durée corrigée est done de 87 jours.

Le cycle le plus long a été de 280 jours, avec 68 jours de temps perdu. La durée corrigée est de 212 jours.

Il est probablement impossible d'obtenir des cycles plus courts. Par contre on doit pouvoir prolonger leur durée, notamment en conservant longtemps des femelles à jeun.

Résistance des larves, nymphes et adultes. - Nous avons fait gorger des larves de 3 à 90 jours après l'éclosion. Elles restent vivantes plus longtemps, jusqu'à 6 mois, si on les garde dans de bonnes conditions, mais, dans ce cas, refusent souvent de se fixer et se dessèchent dans le sac qui les maintient sur l'hôte.

Les nymphes à jeun sont moins résistantes : le plus souvent, elles meurent de 20 à 30 jours après la mue. Nous avons réussi une fois à 
faire gorger des nymphes 65 jours après la mue et une autre fois 33 jours. La période la plus favorable pour le repas est du $1^{\text {er }}$ au $15^{\circ}$ jour après la mue.

En ce qui concerne les adultes à jeun, ils restent vivants plus de 10 mois, si on les tient à l'abri de fa sécheresse.

\section{Durée des diverses phases du cycle}

\section{TABleaU I}

Nota: Dans chaque colonne, les chiffres ordinaires représentent lcs moyennes, fournies par un nombre d'élevages variable selon les mois et les phases. Nous avons élevé au total 51 familles pendant 1 à 4 générations, ce qui représente finalement un nombre d'individus extrêmement grand. Les chiffres sont placés dans l'ordre suivant : Minimum, Maximum, Moyenne.

Les chiffres gras concernent certains résultats exceptionnels, s'écartant par trop des autres pour qu'il puisse en être raisonnablement tenu compte dans l'établissement des moyennes.

Lorsque la durée d'une phase dépasse 30 jours, le chiffre exprimant cette durée est inscrit en face du mois au cours duquel la phase a débuté.

\begin{tabular}{|c|c|c|c|c|c|c|c|}
\hline Mors & $\begin{array}{l}\text { INCUBA- } \\
\text { TION }\end{array}$ & $\begin{array}{l}\text { Repas } \\
\text { LARVE }\end{array}$ & $\begin{array}{l}\text { SOMMEIL } \\
\text { LARVAIRE }\end{array}$ & $\begin{array}{c}\text { REPAS } \\
\text { NYMPHE }\end{array}$ & $\begin{array}{l}\text { SOMMEIL } \\
\text { N Y MPHAL }\end{array}$ & $\begin{array}{c}\text { REPAS } \\
\text { FEMELLE }\end{array}$ & $\begin{array}{l}\text { Gesta- } \\
\text { TION }\end{array}$ \\
\hline Janvier..... & $48-72-65$ & $5-7-6$ & $\begin{array}{c}5-7-6 \\
76\end{array}$ & $7-7-7$ & & 14 & 79 \\
\hline Février..... & & $7-11-9$ & & $6-9-7$ & $\begin{array}{c}10-13-12 \\
108\end{array}$ & $10-15-11$ & $50-90-54$ \\
\hline Mars....... & $31-44-42$ & $5-9-8$ & $\begin{array}{c}10-12-11 \\
62\end{array}$ & & $\cdot$ & $9-13-11$ & $44-54-49$ \\
\hline Avril....... & $38-40-39$ & $7-10-8$ & 37 & $6-6-6$ & & & \\
\hline Mai ........ & $39-40-39$ & $4-6 \cdot 4$ & $10-15-13$ & $5-5-5$ & $26-35-30$ & $9-11-10$ & 50 \\
\hline Juin ........ & $32-47-33$ & 4 & $6-11-8$ & $6-7-7$ & $13-31-20$ & 10 & 11 \\
\hline Juillet..... & $28-36-30$ & $3-7-5$ & 5 & $5-7-6$ & $13-20-15$ & 7-13-9 & $6-15-10$ \\
\hline Août ........ & $28-42-30$ & $4-6 \cdot 5$ & $6-9-7$ & 7 & & $10-15-12$ & $6-14-9$ \\
\hline Septembre. & $30-46-34$ & 5 & 9 & 6 & $31-35-32$ & & \\
\hline Octobre... & $35-46-40$ & $3-6-5$ & $5-8-7$ & 8 & 55 & 12 & $20-40-35$ \\
\hline Novembre.. & $59-79-69$ & $7-5-6$ & $8-14-10$ & $7-9-9$ & $41 \cdot 56 \cdot 50$ & $9-15-13$ & $50-65-60$ \\
\hline Décembre.. & $52-75-60$ & 6 & $9-10-10$ & $8-9-9$ & 33 & 14 & \\
\hline
\end{tabular}


TABLEAU II

\begin{tabular}{|c|c|c|c|c|c|c|}
\hline \multirow[t]{2}{*}{ PÉRIOdE de PONTE } & \multicolumn{3}{|c|}{$\begin{array}{l}\text { DURÉES RÉELLES } \\
\text { MOYENNES DES CYCLES }\end{array}$} & \multicolumn{3}{|c|}{$\begin{array}{l}\text { DURÉEs CORRIGÉES } \\
\text { MOYENNES DES CYCLES }\end{array}$} \\
\hline & Min. & Max. & Moy. & Min. & Max. & Moy. \\
\hline Avril-Août (mois chauds).. & 93 & 148 & 104 & 87 & 103 & 95 \\
\hline froids) $\ldots \ldots \ldots \ldots \ldots \ldots$ & 161 & 280 & 237 & 126 & 212 & 150 \\
\hline
\end{tabular}

Si nous examinons le tableau I, il est à noter :

a) que la durée de chacun des repas, ne varie guère d'un bout de l'année à l'autre : la larve se gorge en 6 jours (extrêmes : 3 et 11 jours) la nymphe, en 7 jours (extrêmes 5 et 9 ), la femelle en 11 jours (extrêmes 7 et 15), à condition qu'elle soit en présence d'un mâle ; dans le cas contraire, on n'obtient pas la fermeture du cycle.

b) que la gestation varie entre 6 et 90 jours.

c) que la durée de l'incubation oscille entre 28 et 79 jours.

d) que, pour ces deux phases, les courtes durées s'observent pendant les mois chauds, les longues pendant les mois froids .

$e$ ) que les périodes de sommeil larvaire et nymphal sont sensiblement plus longues en été qu'en hiver et parfois se prolongent anormalement au cours des mois froids (janvier : 76 jours au lieu de 6 jours ; février : 108 jours au lieu de 12 jours ; mars : 62 jours au lieu de 11 jours).

La durée des cycles est done fonction de la durée de la gestation, de l'incubation et, accessoirement, du sommeil larvaire et nymphal, et nous devons avoir, en été des cycles brefs, en hiver des cycles longs. Cette conclusion se trouve vérifiée par nos élevages ainsi que le montre le tableau II.

Les temps les plus courts ont été obtenus avec des œufs pondus en mai-juin, qui ont permis de fermer le cycle en 3 mois. Avec des œufs pondus en septembre, on a obtenu le cycle le plus long : plus de 9 mois.

Le tableau III donne le détail de trois cycles typiques, dont nous avons accentué le caractère en réduisant ou augmentant les temps perdus. 
TABlEAU III

\begin{tabular}{|c|c|c|c|c|c|c|c|c|c|}
\hline \multirow[b]{2}{*}{ Phases } & \multicolumn{3}{|c|}{ Famille X-1,2 } & \multicolumn{3}{|c|}{ FAMILLE Z-102 } & \multicolumn{3}{|c|}{ FAMHLLE Z-89 } \\
\hline & 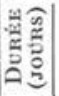 & Mors & Hòte & 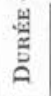 & Mors & HòTE & 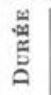 & Mors & Hôte \\
\hline $\begin{array}{l}\text { Incubation ......... } \\
\text { Repas L............ } \\
\text { Sommeil larvaire... } \\
\text { Repas N........... } \\
\text { Sommeil nymphal. } \\
\text { Repas F........... } \\
\text { Gestation.......... } \\
\text { Incubation ......... }\end{array}$ & $\begin{array}{r}40 \\
4 \\
6 \\
5 \\
13 \\
13 \\
6\end{array}$ & $\begin{array}{l}\text { Juin } \\
\text { Juin } \\
\text { Juin } \\
\text { Juil. } \\
\text { Juil. } \\
\text { Juil. } \\
\text { Août }\end{array}$ & $\begin{array}{l}\text { Veau } \\
\text { Veau } \\
\text { Veau }\end{array}$ & $\begin{array}{r}78 \\
9 \\
92 \\
62 \\
7 \\
25 \\
7 \\
7\end{array}$ & $\begin{array}{c}\text { Déc. } \\
\text { Mars } \\
\text { Mai } \\
\text { Juin } \\
\text { Juil. } \\
\text { Juil. } \\
\text { Août }\end{array}$ & $\begin{array}{l}\text { Veau } \\
\text { Lapin } \\
\text { Veau }\end{array}$ & $\begin{array}{r}6 \\
5 \\
8 \\
55 \\
9 \\
90 \\
23\end{array}$ & $\begin{array}{l}\text { Sept. } \\
\text { Sept. } \\
\text { Oct. } \\
\text { Déc. } \\
\text { Mars } \\
\text { Juin } \\
\text { Juil. }\end{array}$ & $\begin{array}{l}\text { Lapin } \\
\text { Lapin } \\
\text { Veau }\end{array}$ \\
\hline $\begin{array}{l}\text { Durée totale cor- } \\
\text { rigée } \ldots \ldots \ldots \ldots \ldots\end{array}$ & & 87 jou & & & 195 joı & urs & & 212 jot & ars \\
\hline $\begin{array}{l}\text { Temps perdu à ajou- } \\
\quad \text { ter } \ldots \ldots \ldots \ldots \ldots \ldots\end{array}$ & & 6 jou & & & 80 jou & & & 68 jot & ars \\
\hline Durée totale vraie.. & & 93 jor & ars & & 275 jo & urs & & 280 jo & urs \\
\hline
\end{tabular}

La tique mère $X-1,2$ avait commencé à pondre à une saison très favorable (mai) et l'éclosion a eu lieu en juin. Il nous a suffi de réduire les temps perdus pour obtenir un cycle estival très bref.

La tique Z-102 a été récoltée sur un chameau au début de l'hiver. L'incubation et le sommeil larvaire ont été très longs. Par ailleurs, nous avons volontairement conservé à jeun la larye pendant 47 jours, la nymphe pendant 23 jours et la femelle pendant 10 jours.

Quant à la tique Z-89, envoyée de province, elle avait pondu en cours de route, nous avons donc commencé à observer le cycle depuis l'éclosion.

Il est intéressant de noter, en comparant Z-102 et Z-89, que les phases prolongées ne sont pas les mêmes. Chez Z-89, le sommeil larvaire et l'incubation sont normaux, tandis que le sommeil nymphal et la gestation sont très longs. Nous avons conservé à jeun la larve pendant 10 jours, la nymphe pendant 8 jours et la femelle pendant 50 jours, ce qui diffère aussi de l'élevage Z-102. 
Alternance. - Si l'on suit plusieurs générations successives de la même famille, on observe une véritable alternance de cycles longs et courts, qui correspondent plus ou moins exactement aux mois froids et aux mois chauds.

Voici, à titre d'exemple (tableau IV), l'alternance observée sur 3 générations de la famille $X-179$.

TABleau IV

\begin{tabular}{|c|c|c|c|}
\hline GÉNÉRATIONS & Début de CyCle & FIN DU CYCLE & DURÉE RÉELLE \\
\hline $\begin{array}{l}\text { I. }(179-3) \ldots \ldots \ldots \ldots \\
\text { II. }(179-3-2) \ldots \ldots \ldots \\
\text { III. }(179-3-2-4) \ldots \ldots \ldots\end{array}$ & $\begin{array}{c}\text { Octobre } \\
\text { Mai } \\
\text { Septembre }\end{array}$ & $\begin{array}{l}\text { Avril } \\
\text { Août } \\
\text { Mai }\end{array}$ & $\begin{array}{l}209 \text { jours } \\
112 \text { jours } \\
272 \text { jours }\end{array}$ \\
\hline
\end{tabular}

Des séries semblables sont la règle dans nos élevages, bien que les tiques soient soustraites aux variations saisonnières normales de la température et de l'humidité. Les différences de durée doivent probablement être plus accentuées dans les conditions naturelles, en raison des froids intenses qui sévissent en hiver.

$\mathrm{Si}$, au laboratoire, il est relativement facile d'obtenir deux générations par an, de septembre à septembre, par exemple, il est douteux que ceci puisse se réaliser fréquemment dans les conditions naturelles.

\section{Conclusions}

1. - Hyalomma dromedarii (Koch 1844) est normalement une tique à 3 hôtes qui, dans certains cas très rares, peut évoluer sur deux hôtes, évitant ainsi des conditions extérieures défavorables.

2. - L'hôte naturel de la larve est inconnu. Les hôtes de prédilection des nymphes et adultes sont, en première ligne, le chameau, en seconde ligne, les bovidés.

3. - Au laboratoire, les larves s'élèvent bien sur lapins et sur veaux, assez mal sur chameaux et moutons. Les nymphes et adultes s'élèvent facilement sur tous ces animaux, mais irrégulièrement sur le mouton. En élevant plusieurs générations sur hôtes de la même espèce, on obtient une véritable adaptation.

4. - Les cycles les plus brefs s'observent en saison chaude et durent au minimum 93 jours. En saison tempérée ou froide, le 
cycle peut durer 280 jours et plus. On peut obtenir deux générations par année avec alternance d'un cycle estival, bref, et d'un cycle hibernal, long.

5. - Les différences, dans la durée des cycles, sont fonction surtout des périodes de gestation et d'incubation, ainsi que des périodes de sommeil larvaire et nymphal. Les repas ont, en toute saison, une durée à peu près constante.

6. - Ces résultats sont basés sur l'étude de 51 familles pures, comprenant chacune de 1 à 4 générations.

\section{Bibliographie (1)}

Brumpt (E.). - Les piroplasmes des bovidés et leurs hôtes vecteurs. Bull. Soc. Path. Exot., XIII, 1920, p. 416-460.

LEwIs (E. A.). - Some tick Investigations in Kenya Colony. Parasitology, XXIV, 1932 , p. 175 .

Lounsbury. - Notes on some South African Ticks. Div. entomol. U.S.A. Bull. 26, n. s. 1900 , p. 41-48; Ticks and African Coast Fever. Transuaal Agric. Journ., Pretoria, II, 1903, p. 4.

Nutrall. - Observations on the biology of Ixodida. Parasitology, VI, 1913, p. 68.

Sergent (Ed.), Donatien, Parrot et Lestoquard. - Transmission naturelle de la theileriose bovine en Afrique du Nord. Arch. I.P. Algérie, IX, 1931, p. 527.

Tonelli-Rondelli. - Miss. Sc. del Prof. Zavattari nel Fezzan. Boll. d. Soc. Entom. Ital., LXIV, 1932, p. 107.

(1) Cette bibliographie ne comprend que les noms d'auteurs cités dans l'article.

Pour la description d'H. dromedarii, màle et femelle, voir : Despy. Notes sur les Ixodidés du genre Hyalomma. Annales de Parasitologie, XIV, 1936 , p. 206.

Pour la description des larves et des nymphes, voir : Delpy. - Description de $H$. dromedarii. Morphologie de la larve et de la nymphe. Annales de Parasitologie, XV, 1937, p. 481-486.

Service des Recherches vétérinaires, Hessarek-Karadj, Téhéran (lran). 